\title{
Requerimiento de cirugías neuro ortopédicas en pacientes con enfermedades neuromusculares que acuden al Hospital de Clínicas, San Lorenzo, Paraguay, desde 2016 al 2018"1
}

\section{Neuroorthopedic surgeries required in patients with neuro- muscular diseases who come to the Hospital de Clínicas, San Lorenzo, Paraguay, from 2016 to 2018}

\author{
Shirley Galeano ${ }^{12}$; Esteban Acosta $^{13}$; Gabriel Cardozo ${ }^{14}$
}

\section{DOI: https://doi.org/10.36003/Rev.investig.cient.tecnol.V2N2(2018)3}

\section{RESUMEN}

Las enfermedades neuromusculares presentan una pérdida de fuerza progresiva, evolucionando hacia una discapacidad severa. Presentan una gran variabilidad en cuanto a severidad, edad de inicio de la sintomatología y las complicaciones. Fue un estudio observacional descriptivo, retrospectivo. Ingresaron al estudio 30 pacientes de ambos sexos con enfermedades neuromusculares. El 70\% de los pacientes evaluados correspondieron al sexo masculino. Edad de $10 \pm 4.7$ años. El $25 \%$ presentaron Distrofia Muscular de Duchenne, el $23 \%$ Atrofia Espinal Tipo II, el $10 \%$ Distrofia Muscular de Becker, el 8\% Distrofia Escapulo Humeral, 4\% Enfermedad de Pompe, 10 \% AME Tipo I y el 20\% con diagnóstico a determinar. El 70\% disnea como principal síntoma, 40\% sin marcha y $3 \%$ escoliosis. Los tipos de curvatura de la columna vertebral en dos pacientes con distrofia muscular de Duchenne fue toracolumbar y mayor ángulo de Cobb, el paciente con Atrofia muscular espinal tipo II fue de tipo lumbar con menor desviación de la columna. Del total de pacientes cinco presentaron marcha tipo I, siete marcha Tipo II, seis marcha Tipo III y doce sin marcha. La cirugía del tendón de Aquiles bilateral requerida por quince pacientes, tres necesitan artrodesis de columna, y varios pacientes requieren de más de dos tipos de cirugías. En Paraguay existe una limitación con respecto a los tipos de cirugías neuroortopédicas posibles de realizar, lo que obliga a muchos pacientes a viajar a países como Argentina o Chile para acceder a las mismas, la artrodesis de columna no se realiza en ninguna institución pública ni privada del país.

Palabras clave: Enfermedades neuromusculares; Artrodesis; Distrofia muscular de Ducheme.

\footnotetext{
${ }^{11}$ Fecha de recepción: noviembre 2018; fecha de aceptación: noviembre 2018

${ }^{12}$ Jefa de Residentes e Internos de la Cátedra y Servicio de Neumología de la Facultad de Ciencias Médicas de la Universidad Nacional de Asunción. Docente de la Cátedra de Anatomía Patológica de la Facultad de Medicina de la Universidad Privada María Serrana. Docente de la Cátedra de Histología II de la Facultad de Medicina de la Universidad Privada María Serrana.

${ }^{13}$ Jefe de Residentes e Internos de la Cátedra y Servicio de Ortopedia y Traumatología de la Facultad de Ciencias Médicas de la Universidad Nacional de Asunción.

${ }^{14}$ Licenciado en enfermería del servicio de Urgencias del Hospital de Clínicas de la Universidad Nacional de Asunción.
} 


\section{ABSTRACT}

Neuromuscular diseases show a progressive loss of strength, evolving towards a severe disability. They present a great variability in terms of severity, age at onset of symptoms and complications. It was an observational, descriptive, retrospective study. Thirty patients of both sexes with neuromuscular diseases were included in the study. $70 \%$ of the patients evaluated corresponded to the male sex. Age of $10 \pm 4.7$ years. $25 \%$ presented Duchenne Muscular Dystrophy, 23\% Spinal Atrophy Type II, 10\% Becker Muscular Dystrophy, 8\% Humeral Scapular Dystrophy, 4\% Pompe Disease, 10\% AME Type I and $20 \%$ diagnosed with decide. $70 \%$ dyspnea as the main symptom, $40 \%$ without gait and $3 \%$ scoliosis. The types of curvature of the spine in two patients with Duchenne muscular dystrophy was thoracolumbar and greater angle of Cobb, the patient with spinal muscular atrophy type II was of lumbar type with less deviation of the spine. Of the total number of patients, five had type I gait, seven gait Type II, six gait Type III and twelve gait without gait. The bilateral Achilles tendon surgery required by fifteen patients, three require spinal fusion, and several patients require more than two types of surgeries. In Paraguay there is a limitation regarding the types of neuro-orthopedic surgeries that can be performed, which forces many patients to travel to countries such as Argentina or Chile to access them, column arthrodesis is not done in any public or private institution from the country.

Keywords: Neuromuscular diseases; Arthrodesis; Duchenne muscular dystrophy 


\section{INTRODUCCIÓN}

Las enfermedades neuromusculares presentan una pérdida de fuerza progresiva, evolucionando hacia una discapacidad severa. Existen más de 150, tipos de enfermedades neuromusculares, para su clasificación generalmente se toma en cuenta la etiología $(1,2)$.

Las distrofias como la Distrofia Muscular de Duchenne (DMD) más común diagnosticada durante la infancia, causada por una mutación en el gen que codifica la distrofina, debido a que la distrofina está ausente, las células musculares se dañan fácilmente. La Distrofia Muscular de Becker (DMB) es menos grave que la de Duchenne y se produce cuando la distrofina se fabrica, pero no en la forma ni cantidad normal. La diferencia principal es que la evolución de la enfermedad es mucho más lenta y es menos común y la distrofia muscular facio-escápulo-humeral (distrofia FEH) es una enfermedad neuromuscular de evolución progresiva en la mayoría de los casos, a veces por brotes, sin afectación de otros órganos más que el músculo esquelético, en el que se manifiesta de forma focal en los músculos de cara, hombros y brazos (2).

La atrofia muscular espinal (AME) es un trastorno hereditario de las neuronas motoras espinales y bulbares que causan atrofia y debilidad musculares, usualmente de forma simétrica y proximal, con predominio de extremidades inferiores y estando respetada la musculatura facial y el intelecto, con sus tres variantes AME Tipo 1 más grave por su comienzo antes de los 6 meses, fallecimiento antes de los 2 años, no se logra la sedestación, AME tipo II con comienzo entre 6 y 18 meses, fallecimiento en la adolescencia, no se logra la deambulación autónoma, marcada atrofia y AME tipo III comienzo después de los 18 meses (hasta los 18 años) (3).

Las de acumulación como la enfermedad de Pompe en la que carecen del ni- vel necesario de una enzima denominada alfa-glucosidasa ácida (GAA) o maltasa ácida. Esta enzima es fundamental para metabolizar adecuadamente el glucógeno. Si no se dispone de la enzima necesaria el glucógeno se acumula en las células musculares, y éstas se pueden dañar tanto que los músculos sufren un deterioro y dejan de responder correctamente (4).

No tienen actualmente un tratamiento etiológico y este aspecto unido a la pérdida de fuerza como principal manifestación clínica, ha desarrollado su manejo en el campo de la rehabilitación, en el que las ortesis y ayudas técnicas adquieren una relevancia predominante. Presentan una gran variabilidad en cuanto a severidad, progresividad, edad de inicio de la sintomatología y edad de aparición de las complicaciones. En este camino que se emprende con el apoyo y compromiso familiar y del paciente, sobrevienen deformidades inherentes a su enfermedad de base que ya no pueden resolverse en forma no invasiva. Los equipamientos (valvas, ortesis, corsés, etc.) no cumplen con su función ya que no logran contener esas deformidades irreductibles y se hace necesaria la cirugía para poder mantener la funcionalidad $(3,4)$.

En la actualidad, se cuenta con pocos recursos terapéuticos medicamentosos y quirúrgicos en el Paraguay, por lo que pacientes y familiares acuden a países vecinos para someterse a las cirugías neuroortopédicas que requieren. Conocer los tipos de cirugías neuroortopédicas que requieren los pacientes con enfermedades neuromusculares que acuden al Departamento de Rehabilitación Cardiorrespiratoria del Hospital de Clínicas de la Facultad de Ciencias Médicas de la Universidad Nacional de Asunción derivados de centros de referencia en el manejo de patologías neuromusculares públicos y privados del país en el periodo del 2016 al 2018, es el objeto de esta presentación $(5,6)$. 


\section{METODO}

El estudio se ejecutó sobre la base de la búsqueda de información primaria en el a través de entrevistas personalizadas y de las historias clínicas de aquellos pacientes con afecciones neuromusculares que consultaron en el Departamento de Rehabilitación Cardiopulmonar de la Carrera de Kinesiología de la Facultad de Ciencias Médicas y evaluados por el servicio de Ortopedia y Traumatología del Hospital de Clínicas de la Universidad Nacional de Asunción desde marzo del 2016 a noviembre del 2018. Fue un estudio observacional descriptivo, retrospectivo.

La población fue enfocada, con 30 pacientes de ambos sexos con enfermedades neuromusculares que acudieron a consulta

Criterios de inclusión:

- $\quad$ Pacientes que acudieron a consulta en al Departamento de Rehabilitación Cardiopulmonar y evaluados por el servicio de Ortopedia y Traumatología del Hospital de Clínicas de la Universidad Nacional de Asunción.

- Ambos sexos.

- Pacientes que poseían diagnóstico confirmado/sospecha de enfermedad neuromuscular.

- $\quad$ Poseían registrada su historia clínica correspondiente en los archivos del Hospital de Clínicas.
- Pacientes que acudieron a la consulta desde marzo del 2016 hasta noviembre del 2018.

Criterios de exclusión:

- Historias clínicas de pacientes que no poseían diagnóstico de enfermedad neuromuscular.

- Consultas que no poseían registrada su historia clínica correspondiente en los archivos del Hospital de Clínicas.

- Pacientes que acudieron fuera del marco temporal preestablecido.

Los datos recogidos fueron analizados y procesados utilizando el programa informático Microsoft Excel. Los resultados se presentan en tablas y gráficos.

Aspectos éticos:

Anonimato: no se revelará el nombre de ningún paciente que haya participado de la recolección de datos, para de esta manera proteger la identidad y privacidad de quienes colaboraron con el proceso investigativo.

Criterio de justicia y beneficencia: es un criterio de no maleficencia, en el cual no se publicarán los datos, sino que solamente se darán a conocer los resultados finales dentro del derecho de anonimato de los participantes.

Se consideran las siguientes variables: sexo, edad, tipo de enfermedad neuromuscular, signos y síntomas, tipos de marcha y tipos de curvatura de la columna vertebral.

\section{RESULTADOS}

El $70 \%$ de los pacientes evaluados correspondieron al sexo masculino. (Gráfico 1) Promedio de edad de $10 \pm 4.7$ años. El 25 $\%$ presentaron Distrofia Muscular de Duchenne, el $23 \%$ Atrofia Espinal Tipo II, el $10 \%$ Distrofia Muscular de Becker, el 8\% Distrofia Escapulo Humeral, 4\% Enfermedad de Pompe, $10 \%$ AME Tipo I y el 20\% con diagnóstico a determinar. (Gráfico 2) El $70 \%$ presentaron disnea como princi- pal síntoma, $40 \%$ no presentaron marcha y 3\% tienen escoliosis. (Gráfico 3) Los tipos de curvatura de la columna vertebral en dos pacientes con distrofia muscular de Duchenne fue toracolumbar y mayor ángulo de Cobb, el paciente con Atrofia muscular espinal tipo II fue de tipo lumbar con menor desviación de la columna. (Gráfico 4) Del total de pacientes cinco presentaron marcha tipo I, siete marcha 
Tipo II, seis marcha Tipo III y doce sin ce pacientes, tres necesitan artrodesis de marcha. (Gráfico 5). La cirugía del tendón columna, y varios pacientes requieren de de Aquiles bilateral requerida por quin- más de dos tipos de cirugías. (Gráfico 6)

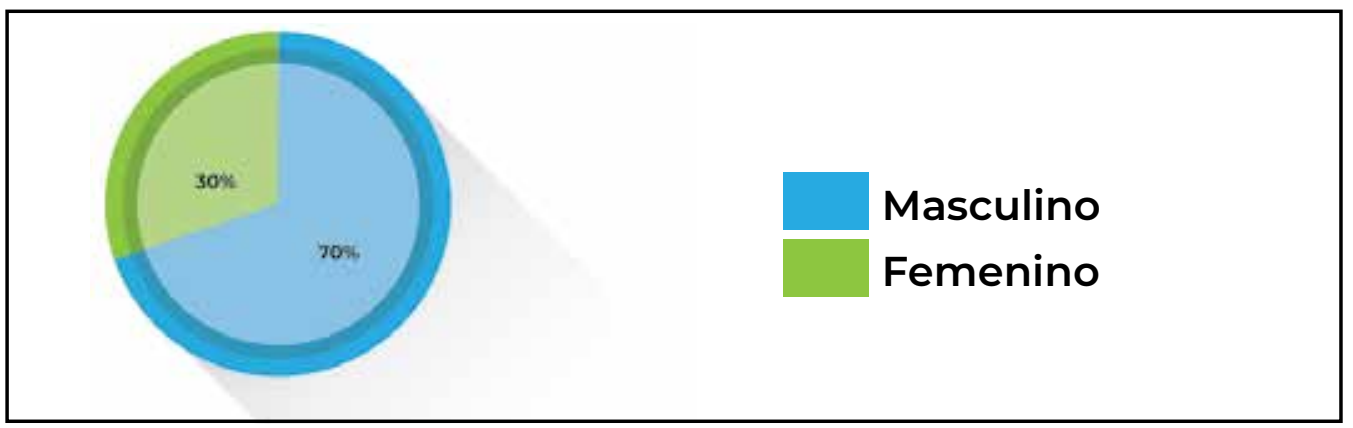

Gráfico 1. Distribución por sexo. Pacientes con ENM. Hospital de Clínicas. 2016-2018

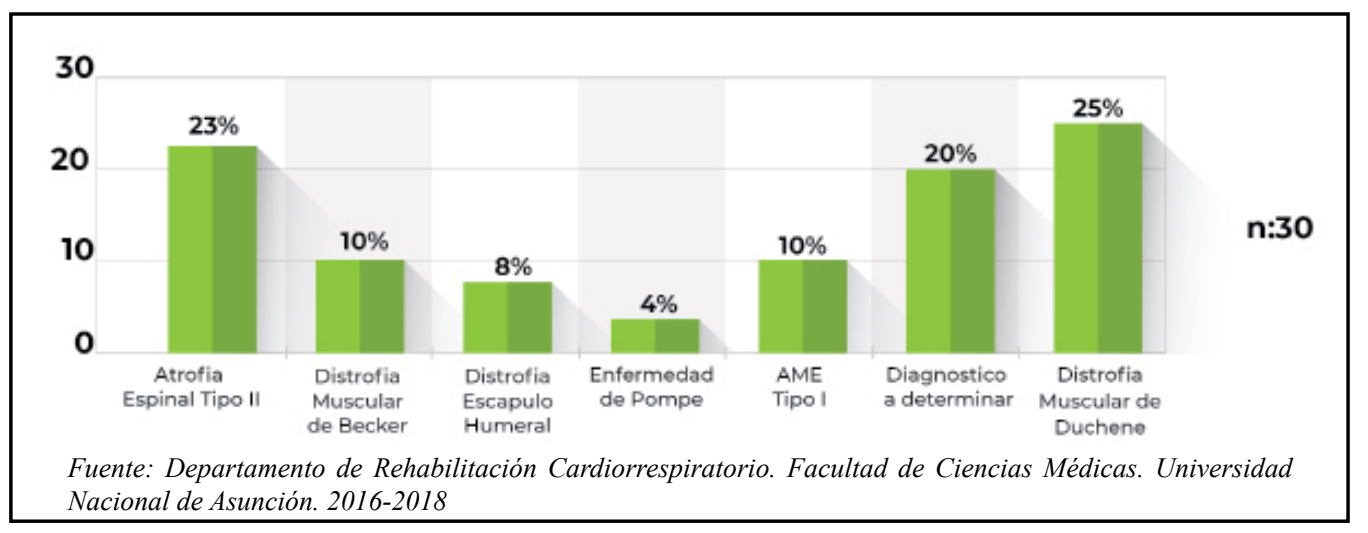

Gráfico 2. Tipo de Enfermedad Neuromuscular. Pacientes con ENM. Hospital de Clínicas.2016-2018

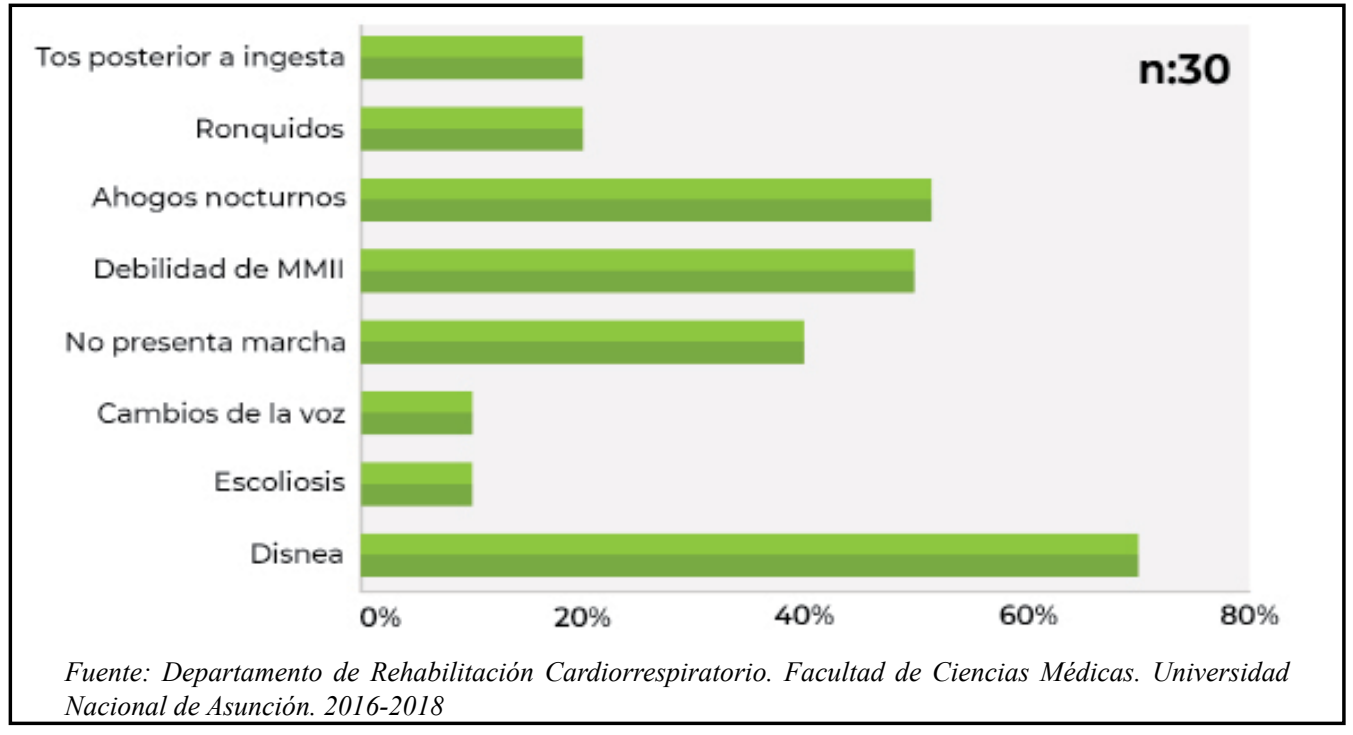

Gráfico 3. Signos y síntomas. Pacientes con ENM. Hospital de Clínicas.2016-2018. 


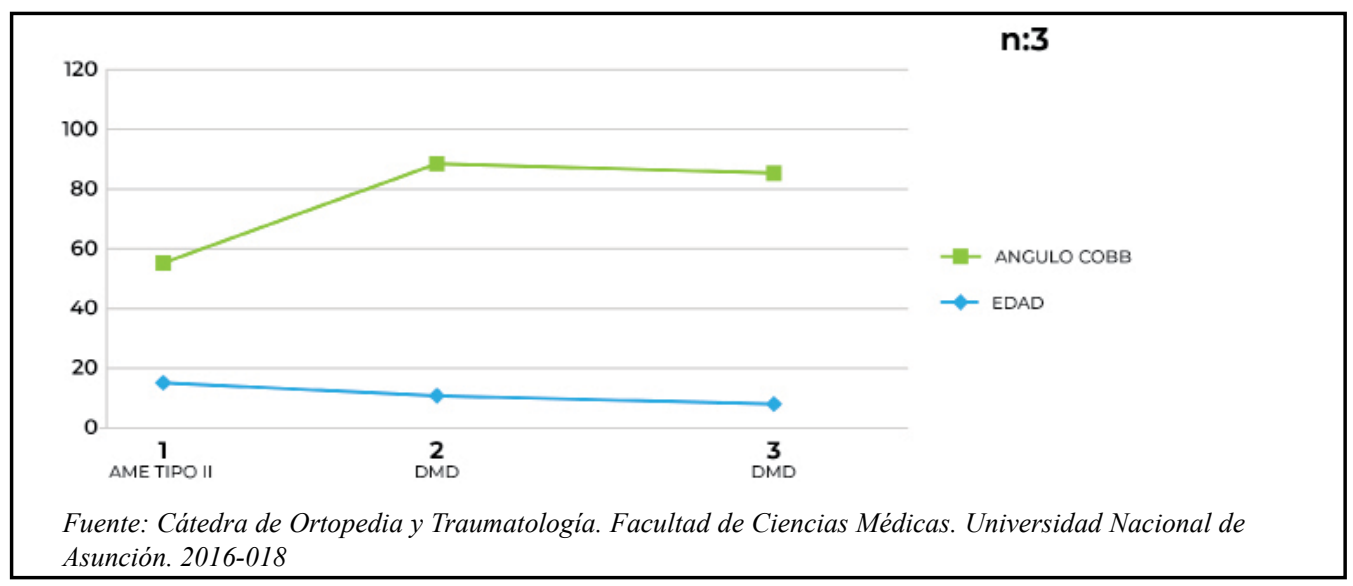

Gráfico 4. Tipo de curvatura de la columna vertebral. Pacientes con ENM. Hospital de Clínicas. 2016-2018

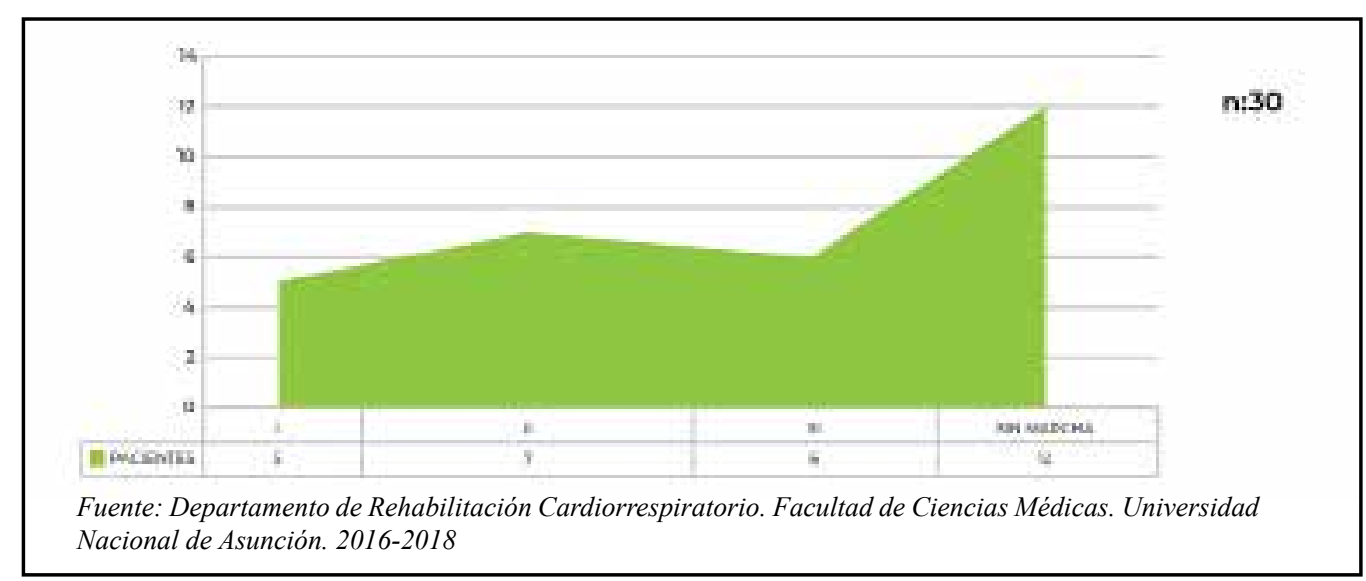

Gráfico 5. Tipo de Marcha según Schuterland. Pacientes con ENM. Hospital de Clínicas.2016-2018.

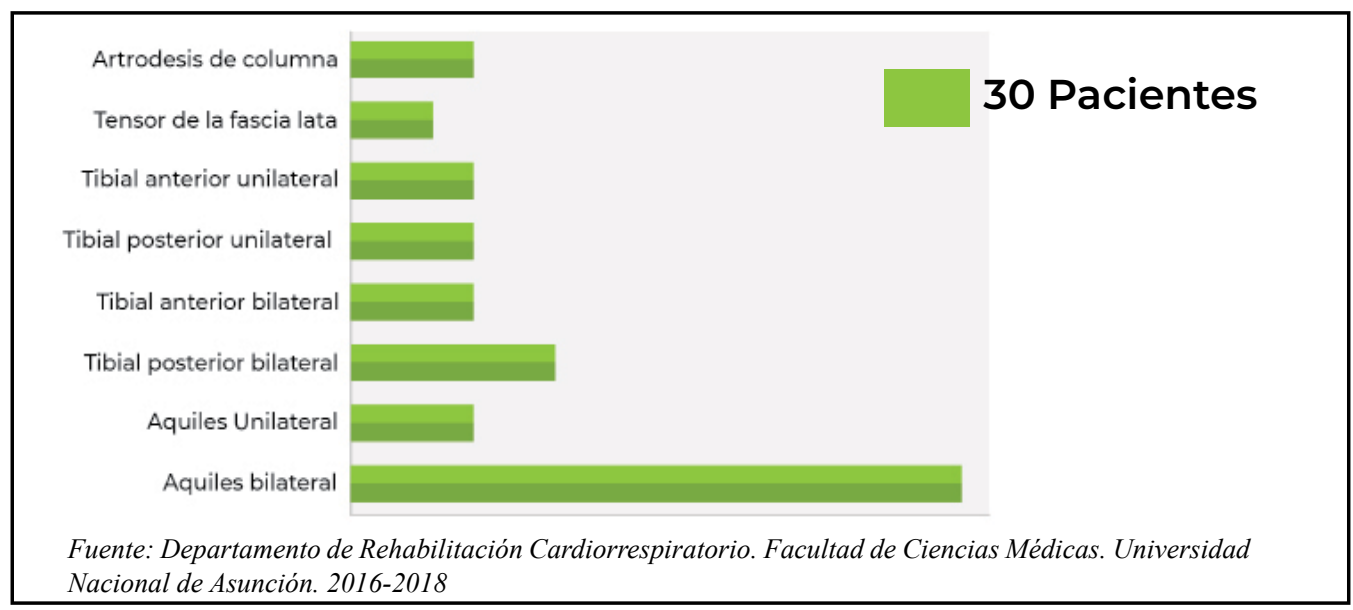

Gráfico 6. Cirugías Neuroortopedicas. Pacientes con ENM. Hospital de Clínicas.2016-2018. 


\section{DISCUSIÓN}

La distrofia muscular progresiva de $\mathrm{Du}-$ chenne es una enfermedad muscular de progresión, hasta la fecha inexorable. La parálisis miógena progresiva lleva a estos pacientes a la cuadriplejía y al derrumbamiento de su raquis con la aparición de deformidades que dan incomodidad al paciente y favorecen la presencia de patologías agregadas $(7,8)$.

Si nos encontramos ante un paciente en el primer estadio de marcha (Sutherland), la indicación de una ortesis y la kinesioterapia podrían bastar para mejorar la estabilidad del niño durante la bipedestación y marcha; si el paciente presenta ya un tipo II o III de Sutherland, será el momento de pensar en la cirugía, para evitar que las posiciones viciosas consecuentes a desequilibrios musculares, impidan su relativa independencia $(9,10,11)$.
Similar a este estudio, el grupo español de Arendar et al, informan que 20 pacientes con Distrofia Muscular Progresiva, fueron sometidos a intervenciones quirúrgicas varias, siendo los músculos más frecuentemente abordados el complejo Aquíles/ Aquiles-soleo, el tibial posterior, el tibial anterior y el tensor de la fascia lata. En todos los casos se siguió el plan: "corta inmovilización, pronta movilización” (12).

En nuestro país existe una limitación con respecto a los tipos de cirugías neuroortopédicas posibles de realizar, lo que obliga a muchos pacientes y familiares a viajar a países como Argentina o Chile para acceder a las mismas, la artrodesis de columna no se realiza en ninguna institución pública ni privada del Paraguay.

\section{CONCLUSIONES}

E1 $25 \%$ presentaron Distrofia Muscular de Duchenne, el $23 \%$ Atrofia Espinal Tipo II, el $10 \%$ Distrofia Muscular de Becker, el 8\% Distrofia Escapulo Humeral, 4\% Enfermedad de Pompe, $10 \%$ AME Tipo I y el $20 \%$ con diagnóstico a determinar. El 70\% presentaron disnea como principal síntoma, $40 \%$ no presentaron marcha y $3 \%$ tienen escoliosis. Los tipos de curvatura de la columna vertebral en dos pacientes con distrofia muscular de Duchenne fue toracolumbar y mayor ángulo de Cobb, el paciente con Atrofia muscular espinal tipo II fue de tipo lumbar con menor desviación de la columna. La cirugía del tendón de Aquiles bilateral requerida por quince pacientes, tres necesitan artrodesis de columna, y varios pacientes requieren de más de dos tipos de cirugías.

\section{BIBLIOGRAFÍA}

1. Deenen JC, Horlings CG, Verschuuren JJ, Verbeek AL, van Engelen BG. The Epidemiology of Neuromuscular Disorders: A Comprehensive Overview of the Literature. J Neuromuscul Dis. 2015;2(1):73-85.

2. Salinas $P$ et al. Cuidados Respi- ratorios para pacientes con Enfermedades Neuromusculares. Rev. Neumol Pediatr 2017; 12(3): 103-113.

3. Bach JR, Gonçalves MR, Hamdani I, Winck JC. Extubation of patients with neuromuscular weakness: a new management paradigm. Chest. 2010 May; 
137(5):1033-9.

4. Bach JR. Conventional approaches to managing neuromuscular ventilatory failure. Ed. Pulmonary rehabilitation: the obstructive and paralytic conditions. Philadelphia, PA: Hanley \& Belfus, 1996; 285-301.

5. Tzeng AC, Bach JR. Prevention of pulmonary morbidity for patients with neuromuscular disease. Chest. 2000 Nov 118 (5):1390-6.

6. Martínez C, Villa JR, Enfermedad neuromuscular: evaluación clínica y seguimiento, Madrid, España.2014

7. Salinas P, Prado F, Giménez G, Bach J R, Herrero V. Cuidados respiratorios en pacientes neuromusculares. Neumología Pediatrica Cl. Chile, 2017(3):103 -113 .

8. Arendar G, Canelo S, Paladino D. Cirugía de los miembros inferiores en la distrofia muscular progresiva "Enfermedad de Duchenne".Rev. Asoc. Arg. Ortop y Traumatol, Vol58, No 3, pag 286-293.
9. Arendar G, Canelo S, Paladino D. Cirugía funcionalizadora en la distrofia muscular progresiva (Enfermedad de Duchenne. Rev Esp Cir Osteoart 1995; 30: 29-33

10. Curbelo P, Silveri C, Cuneo A, Olivera R. Funcionalidad y calidad de vida luego de la cirugía de escoliosis neuromuscular. Instituto de Ortopedia y Traumatología, Universidad de la República, Uruguay 2017

11. Canelo S. Cirugías más frecuentes en neuromusculaes. Revista Seguir Andando. $\mathrm{N}^{\mathrm{o}} 11.2012$.

12. Arendar G, Canelo S, Paladino D. Cirugía neuroortopedica en la distrofia muscular progresiva. Rev Esp Cir Osteoart 2010; 20: 15-22 